\title{
Verification of Tether Deployment System aboard CubeSat through Dynamics Simulations and Tests
}

\section{Sakamoto, Hiraku}

IEEE

2021

Sakamoto , H, Mughal, M R , Slavinskis , A, Praks , J, Toivanen , P , Janhunen , P , Palmroth , M , Kilpua , E \& Vainio , R 2021, Verification of Tether Deployment System aboard CubeSat through Dynamics Simulations and Tests . in 2021 IEEE AEROSPACE CONFERENCE (AEROCONF 2021) . IEEE Aerospace Conference Proceedings, IEEE, IEEE Aerospace Conference (AeroConf) , 06/03/2021 . https://doi.org/10.1109/AERO50100.2021.9438281

http://hdl.handle.net/10138/339987

https://doi.org/10.1109/AERO50100.2021.9438281

unspecified

acceptedVersion

Downloaded from Helda, University of Helsinki institutional repository.

This is an electronic reprint of the original article.

This reprint may differ from the original in pagination and typographic detail.

Please cite the original version. 


\section{Verification of Tether Deployment System aboard CubeSat through Dynamics Simulations and Tests}

\author{
Hiraku Sakamoto \\ Tokyo Institute of Technology \\ 2-12-1 Ookayama, Meguro \\ Tokyo 152-8552 Japan \\ sakamoto.h.aa@m.titech.ac.jp
}

Petri Toivanen, Pekka Janhunen

Finnish Meteorological Institute

P.O.Box 503, FI-00101 Helsinki, Finland

petri.toivanen@fmi.fi, pekka.janhunen@fmi.fi

\author{
Muhammad Rizwan Mughal, Andris Slavinskis, Jaan Praks \\ Aalto University \\ Maarintie 8, 02150 Espoo, Finland
}

rizwan920@gmail.com, andris.slavinskis@aalto.fi,jaan.praks@aalto.fi

Minna Palmroth, Emilia Kilpua

University of Helsinki

P.O. Box 68, FI-00014 Helsinki, Finland

minna.palmroth@helsinki.fi, emilia.kilpua@helsinki.fi

\section{Rami Vainio \\ Department of Physics and Astronomy \\ 20014 University of Turku, Finland \\ rami.vainio@utu.fi}

\begin{abstract}
This paper proposes a proper model selection strategies for the dynamic simulations of the tether deployment mission aboard a CubeSat. Space tether technology will enable innovative space missions in the near future. The Coulomb Drag Propulsion (CDP), including electric solar wind sailing, is one of the plausible future technologies. The authors currently develop a CubeSat, FORESAIL-1, for space demonstration of CDP. However, the analytical simulations for the verification and validation of the mission design typically require a high computational cost. This is because a minimum model order is not selected properly. In this study, through observing a preliminary analytical model for tether deployment analysis, the simplest model is chosen to avoid the mission failure modes in each deployment phase.
\end{abstract}

\section{INTRODUCTION}

Space tether is one of the enabling mechanical systems that will realize innovative space missions in the near future. Such space missions include capturing of space debris or small celestial bodies using tethers [1]; electrodynamic tether systems for orbit transfer [2]; construction of space elevator [3]; and Electric solar wind sail (E-sail) propulsion using charged tethers [4], [5], as illustrated in Fig. 1. However, although there are substantial numbers of research papers on the theories and numerical simulations on the dynamics of space tethers, only a small number of technology demonstrations have been implemented in space yet. One of the reasons is that most tether systems are extremely large and flexible; thus the verification of their design before the flight is very challenging.

The use of CubeSats is one plausible option to realize innovative technology demonstration of tether systems in space since they are developed and launched at a much lower cost than conventional satellites. The authors of this paper currently develop $3 \mathrm{U}$ CubeSat FORESAIL-1 to realize the world's first space demonstration of Coulomb drag propulsion [6], [7]. FORESAIL-1 will demonstrate the plasma brake for deorbiting in the Low Earth Orbit (LEO). The same technology can be used for the E-sail for interplanetary exploration since both technologies employ the Coulomb interaction between a charged tether and a plasma flow. The previous attempts of ESTCube-1 [8] and Aalto-1 [9], [10] to demonstrate the CDP in orbit have been limited in success, emphasizing the importance of this paper. Although the satellite is as small as $4 \mathrm{~kg}$, a $300 \mathrm{~m}$-length tether will be deployed. As a result, testing the mission sequences for the tether deployment on the ground is almost impossible. The verification of the design inevitably involves analytical dynamic simulations.

However, it is not clear what kinds of analyses will enable efficient verification. The possible analytical models range from a single rigid-body model; a rigid-body with pendulumlike tip masses [11], [12]; an articulated rigid bar model for tether flexibility representation; a spring-mass model that considers both axial and bending deformations; to a highfidelity finite-element model. A proper selection criteria for suitable modeling to verify the mechanical and controller designs has rarely discussed yet.

The verification for dynamic deployment of flexible space structures have been widely studied especially in the spacecraft structure community [13]. There are some past examples of large flexible deployable structures, which were demonstrated on orbit. The dynamic analyses are integrated in design and verification processes, but typically they are time-consuming [13]. One typical example is the numerical

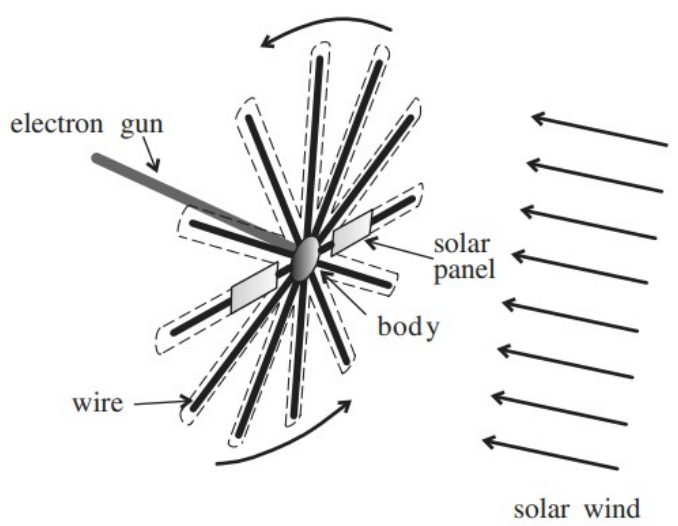

Figure 1. One example of electric solar wind sail architecture. Source: Adapted from [5]. 
dynamic deployment simulations of the solar power sail demonstrator IKAROS [14], [15]. For the dynamic analysis of a $14 \mathrm{~m}$-by-14 m-size square sail deployment, two kinds of models were used: a high-fidelity finite-element model and a low-fidelity spring-mass model. But even with the lowfidelity model, the required computational time for predicting a deployment sequence was approximately 10 hours. This may be too long during a small satellite design phase since the design and verification period typically finishes within only a few months in a CubeSat development.

This problem is caused by the difference in the objectives of the dynamic simulations between conventional theoretical studies and small satellite developments. In conventional studies, the objective is primarily finding new physical insights underlying the nonlinear dynamic behavior of highly flexible structures in space. On the other hand, in small satellite development, the objective is to evaluate a current satellite design as rapidly as possible so that an engineer can build another improved mechanical prototype right after the simulation. The simplest model for the simulation purpose is important for this objective.

Failure Modes and Effects Analysis (FMEA) is often used to improve the design of satellites during the prototyping phase. In other words, the dynamic simulation methods are required to be at least accurate enough to enable the evaluation during the FMEA. Too high accuracy is not desired here because such simulations delay the rapid development of small satellites.

To this end, this paper proposes a method to properly select a dynamic simulation model for deployment analyses of a flexible space tether among several possible modeling options. The plasma brake mission by the $3 \mathrm{U}$ CubeSat FORESAIL1 is used as an example. The development of FORESAIL1 is currently at the transition from the prototyping phase to the Engineering Qualification Model (EQM) integration phase; thus the rapid design verification is actually needed. This paper uses the modeling selection for each plausible failure modes, based on the frequency range that dominates the failure mode dynamics.

The rest of the paper is organized as follows. In $\S 2$, the plasma brake mission, which involves tether deployment, is explained to clarify the analysis objectives. Then in $\S 3$, a simple analytical model for the tether deployment mission is constructed to calculate the frequency ranges appear in the deployment dynamics. In $\$ 4$, a proper modeling method is chosen for a failure mode based on the previous frequency domain analysis. Finally, $§ 5$ concludes the paper.

\section{DESIGN OF TETHER DEPLOYMENT Mission PAYloAd}

This subsection explains the FORESAIL-1 CubeSat design and the plasma brake mission payload aboard the CubeSat, which involves tether deployment and high-voltage charge on the tether.

\section{FORESAIL-1}

FORESAIL-1 is the first in the FORESAIL mission series developed by the Finnish Centre of Excellence for Sustainable Space [7]. The FORESAIL-1 layout is shown in Fig. 2. The satellite is planned to be launched into LEO in 2021. Two major mission payloads are aboard, and one of them is the Plasma Brake mission payload, which demonstrates Coulomb

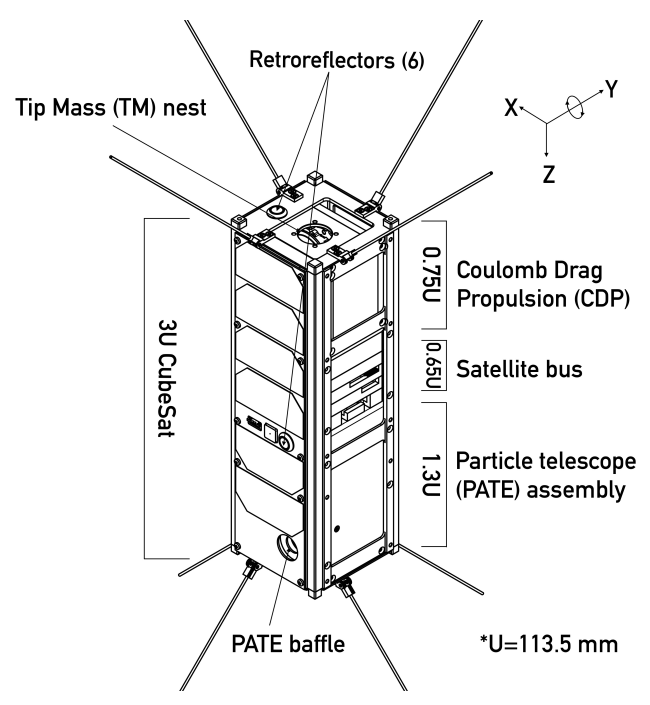

Figure 2. 3U CubeSat FORESAIL-1, currently under development. Source: Adapted from [6].

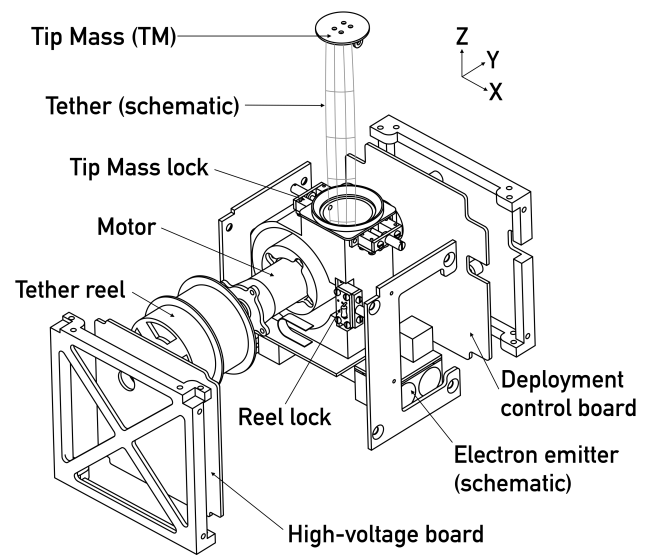

Figure 3. Plasma Brake mission payload. Source: Adapted from [6].

Drag Propulsion (CDP).

\section{Plasma Brake mission payload}

The mission payload hardware is depicted in Fig. 3. This Plasma Brake system deploys a $300 \mathrm{~m}$-length conducting tether. The tether is made of aluminum, and made of $25 \mu \mathrm{m}$ diameter multiple wires bonded together to provide redundancy against cutting [16]. The entire satellite spins and the tether is deployed by centrifugal force in the $-Z$ direction in Fig. 2. The tether is reeled-out using a motor. The spin-up is realized by magnetic torquers on the platform.

Once the tether deployment is completed, the tether is charged with $-1 \mathrm{kV}$ voltage. At this voltage, the expected nominal $\mathrm{PB}$ thrust per tether length is $58 \mathrm{nN} / \mathrm{m}$ when the tether is perpendicular to the ram flow. Figure 4 illustrates the operating principle of the mission. Using the thrust force, FORESAIL-1 will decrease the orbital altitude to demonstrate the nano-satellites' active deorbiting.

The mission sequence of the Plasma Brake mission is described as follows.

1. The launch lock for the tip mass, which weighs about 


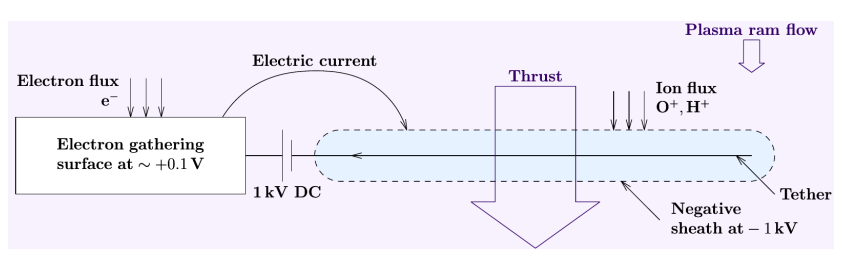

Figure 4. Operating principle of Coulomb drag propulsion. Source: Adapted from [7].

$2.5 \mathrm{~g}$, is released.

2. The satellite is spun up using magnetic torquers in order to provide the centrifugal force which keeps the tether under tension.

3 . The tether is reeled out using a motor very slowly. As the tether is deployed, the spin rate decreases because of the increase of the moment of inertia.

4. Before the system loses the spin stability, the satellite is spun up again. Since the magnetic torqures' actuation does not have high accuracy, the system will experience wobbling motions.

5. Repeating the procedure, the tether deployment is completed.

6. The tether is negatively charged, and then the satellite's attitude and altitude are controlled by Coulomb drag forces.

Since the mission is significantly coupled with the attitude determination sensors and the attitude actuators, the closedloop dynamic response analysis of the satellite during and after tether deployment is important. For the validation and verification of the design, a series of simulations will be conducted with variable design parameters, variable mission sequences, and variable initial conditions with variable environmental effects, including Coulomb drag generation and atmospheric drag. Thus, analytical models are required to be as simple as possible to enable the rapid and iterative design evaluation process.

\section{Frequency Domain ConSIDERATION OF SPACE TeTHER DYNAMICS}

This section derives the simple analytical dynamic model for FORESAIL-1 Plasma Brake tether deployment mission. Then the tether deployment dynamics are analyzed in a frequency domain to enable proper model selections in the next section.

\section{Preliminary Tether Dynamic Model}

The equations of motion for the analytical model, shown in Fig. 5, are derived to understand the basic dynamic behaviors of the tether deployment by the spinning satellite.

The dynamic equations are derived based on the coordinate system at the center of mass $(\mathrm{CoM})$ of the satellite. For generalization, the equations of motion for a spinning satellite with $N$ tethers are derived; and it is reduced to the case of $N=1$ in the next subsection. In this formulation, only the tip mass motion is considered and the mass of the tether is ignored. In addition, the tethers are assumed to be always in taut. In other words, the tension in the tether $i$ is assumed to be always not negative, $T_{i} \geq 0$ where $i=1, \ldots, N$.

Then the vector from the geometric center to the tether deploying point for $i$-th tether, $\boldsymbol{b}_{i}$, and the direction vector from the $i$-th deploying point to the corresponding tip mass, $\hat{c}_{i}$ are written as follows.

$$
\begin{aligned}
& \boldsymbol{b}_{i}=\left[\begin{array}{c}
R \\
y_{i} \\
z_{i}
\end{array}\right] \\
& \hat{\boldsymbol{c}}_{i}=\left[\begin{array}{c}
\cos \left(\phi_{i}\right) \cos \left(\theta_{i}\right) \\
-\cos \left(\phi_{i}\right) \sin \left(\theta_{i}\right) \\
\sin \left(\phi_{i}\right)
\end{array}\right]
\end{aligned}
$$

Using the above expressions, the position of each tip mass from the satellite's CoM is given as

$$
\boldsymbol{r}_{i}=\boldsymbol{r}_{0}+\boldsymbol{b}_{i}+\ell_{i} \hat{\boldsymbol{c}}_{i}
$$

where $\ell_{i}$ is the deployed length of $i$-th tether, $\boldsymbol{r}_{0}$ is the position vector of the satellite's geometric center.

The CoM position of the entire system is

$$
\boldsymbol{r}_{c m}=\frac{1}{M} \sum_{i=1}^{N} m_{i} \boldsymbol{r}_{i}
$$

where $M=m_{b}+\sum_{i=1}^{N} m_{i}$ is the total mass in the system. $m_{b}$ is the mass of the satellite body.

With respect to the CoM of the entire system, the position vectors of the satellite's CoM and the tether tips are $-\boldsymbol{r}_{\mathrm{cm}}$ and $\boldsymbol{r}_{i}-\boldsymbol{r}_{c m}$, respectively. Additionally, since the body-fixed frame rotates with the angular velocity of $\boldsymbol{\omega}=\left[\omega_{1} \omega_{2} \omega_{3}\right]^{T}$, the velocities and the accelerations of the satellite CoM, $\left(\boldsymbol{v}_{b}, \boldsymbol{a}_{b}\right)$ and the $i$-th tether tips, $\left(\boldsymbol{v}_{i}, \boldsymbol{a}_{i}\right)$, have the form of

$$
\begin{aligned}
\boldsymbol{v}_{b}= & -\dot{\boldsymbol{r}}_{c m}+\boldsymbol{\omega} \times\left(-\boldsymbol{r}_{c m}\right) \\
\boldsymbol{v}_{i}= & \left(\dot{\boldsymbol{r}}_{i}-\dot{\boldsymbol{r}}_{c m}\right)+\boldsymbol{\omega} \times\left(\boldsymbol{r}_{i}-\boldsymbol{r}_{c m}\right) \\
\boldsymbol{a}_{b}= & -\ddot{\boldsymbol{r}}_{c m}+\dot{\boldsymbol{\omega}} \times\left(-\boldsymbol{r}_{c m}\right)+2 \boldsymbol{\omega} \times\left(-\dot{\boldsymbol{r}}_{c m}\right)+ \\
& \boldsymbol{\omega} \times\left\{\boldsymbol{\omega} \times\left(-\boldsymbol{r}_{c m}\right)\right\} \\
\boldsymbol{a}_{i}= & \ddot{\boldsymbol{r}}_{i}-\ddot{\boldsymbol{r}}_{c m}+\dot{\boldsymbol{\omega}} \times\left(\boldsymbol{r}_{i}-\boldsymbol{r}_{c m}\right) \\
& +2 \boldsymbol{\omega} \times\left(\dot{\boldsymbol{r}}_{i}-\dot{\boldsymbol{r}}_{c m}\right)+\boldsymbol{\omega} \times\left\{\boldsymbol{\omega} \times\left(\boldsymbol{r}_{i}-\boldsymbol{r}_{c m}\right)\right\}
\end{aligned}
$$

Finally, the equations of translational motion are

$$
\begin{aligned}
m_{b} \boldsymbol{a}_{b} & =\sum_{i=1}^{N} T_{i} \hat{\boldsymbol{c}}_{i} \\
m_{i} \boldsymbol{a}_{i} & =-T_{i} \hat{\boldsymbol{c}}_{i}
\end{aligned}
$$

where $T_{i}$ is the tension in the $i$-th tether. On the other hand, the equations of rotational motion are

$$
\left.I \dot{\boldsymbol{\omega}}+\boldsymbol{\omega} \times(I \boldsymbol{\omega})=\sum_{i=1}^{N}\left\{\left(\boldsymbol{r}_{0}+\boldsymbol{b}_{i}\right) \times T_{i} \hat{\boldsymbol{c}}_{i}\right)\right\}+\boldsymbol{\tau}
$$

where $\boldsymbol{\tau}=\left[\tau_{1} \tau_{2} \tau_{3}\right]^{T}$ is the attitude actuator torques. $\boldsymbol{I}$ is the inertia matrix of the satellite.

Now that the dynamic equations are obtained, the responses can be computed using a numerical integration scheme for variable tether length, $\ell_{i}$, with proper initial conditions. But the current objective is to understand the basic response characteristics. Thus the equations of motion is simplified with several drastic assumptions. 


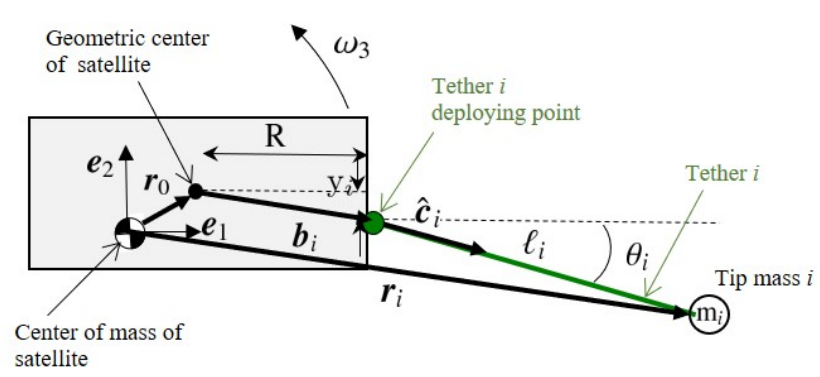

a) Top view (seeing in $-\boldsymbol{e}_{3}$ direction)

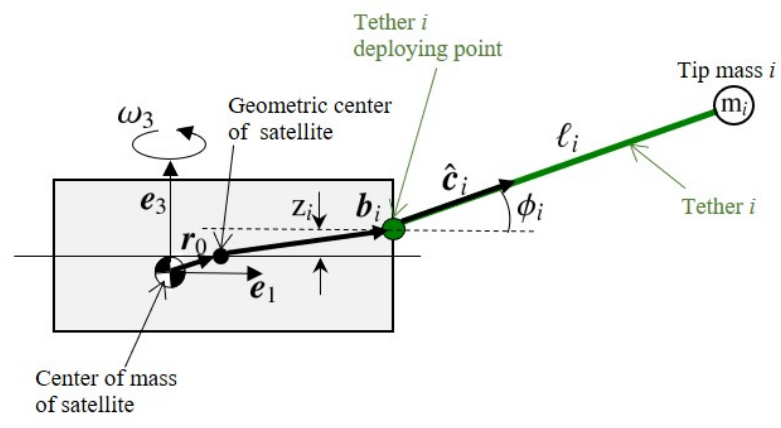

b) Side view (seeing in $\boldsymbol{e}_{2}$ direction)

Figure 5. Preliminary analytical model for FORESAIL-1 tether dynamics.

\section{Single Tether System}

Let $N=1$, and also the following simple case is considered: $\boldsymbol{r}_{0}=\mathbf{0}, \boldsymbol{b}_{1}=\left[\begin{array}{lll}R & 0 & 0\end{array}\right]^{T}$ and small angles are assumed: $\theta_{1} \approx 0$, $\phi_{1} \approx 0$, and $\omega_{1} \omega_{2} \approx 0$. Then $\hat{\boldsymbol{c}}_{1}=[1-\theta \phi]^{T}$. In addition, the following inertia matrix is assumed.

$$
\boldsymbol{I}=\left[\begin{array}{ccc}
I_{1} & 0 & 0 \\
0 & I_{2} & 0 \\
0 & 0 & I_{3}
\end{array}\right]
$$

And the actuator torques are zero, $\boldsymbol{\tau}=\mathbf{0}$.

With this simplification, the equations of rotational motion in Eq. (7) reduces to the following expressions.

$$
\begin{aligned}
I_{1} \dot{\omega}_{1}-\left(I_{2}-I_{3}\right) \omega_{3} \omega_{2} & =0 \\
I_{2} \dot{\omega}_{2}-\left(I_{3}-I_{1}\right) \omega_{3} \omega_{1} & =-R \phi T_{1} \\
I_{3} \dot{\omega}_{3} & =-R \theta T_{1}
\end{aligned}
$$

These equations clarifies the basic characteristics of this motion.

- Equation (9), the rotational motion around $\boldsymbol{e}_{1}$, shows the torque free rotational motion of a rigid-body without any interaction with tether dynamics.

- In Eq. (10), the rotational motion around $\boldsymbol{e}_{2}$, the satellite's rotational motion couples with the out-of-plane swing angle of the tip mass.

- Equation (11) shows the interaction between the spin rate, $\omega_{3}$, and the in-plane swing angle of the tether.

- If the out-of-plane swing motion is small, $\phi \approx 0$, the satellite's rotational motion around the $e_{1}$ and $e_{2}$ axes are the same with the rigid-body dynamics of the satellite before tether deployment. As found in a textbook [17], the characteristic roots, $s$, are

$$
s= \pm j \omega_{3} \sqrt{\frac{\left(I_{2}-I_{3}\right)\left(I_{1}-I_{3}\right)}{I_{1} I_{2}}}
$$

where $j=\sqrt{-1}$.

Next, the vibration frequencies of the tether swing motions can be found from Eq. (6) by ignoring nutation motion of the satellite as $\omega_{1}=\omega_{2}=0$. More precise responses can be obtained by numerical integration when they are needed. In addition, please note that Eq. (5) is linearly dependent on the other equations. Equation (6) yields

$$
\mu\left[2 \omega_{3} \ell_{1} \dot{\theta}-\omega_{3}^{2}\left(R+\ell_{1}\right)\right]=-T_{i}
$$

where

$$
\mu=\frac{m_{b} m_{1}}{m_{b}+m_{1}}
$$

Equation (13) gives the tension in the tether.

Then also from Eq. (6), the out-of-plane swing motion is

$$
\mu \ell_{1} \ddot{\phi}_{1}+T_{1} \phi_{1}=0
$$

This is the undampled single degree-of-freedom vibration, whose natural frequency is $\Omega_{\phi}=\sqrt{\frac{T_{1}}{\mu \ell_{1}}}$.

Thus the dynamic equations are the combination of the nutation motion of the satellite and the swing motions of the tether. In addition, the actual dynamics also involve the following two motions.

- The axial elastic deformation of the tether made of aluminum.

- The transverse higher-mode deformation of the tether due to the distributed mass along the tether length.

\section{Proper Model Selection for Failure MODE ANALYSIS}

The critical failure mode is the instability of the spin dynamics during the spinning up sequence using magnetic torquers. We can naturally assume that the control bandwidth for the magnetic torquers always stays lower than the fundamental natural frequency of the flexible system. In other words, no rapid maneuvers will be implemented for this highly flexible system. This basic operation rules can avoid spillover instability due to the actuation [18].

With this actuation rule, the system motion is then dominated by the fundamental natural frequency of the system. Based on the discussion in the previous section, three kinds of simple models are needed for attitude stability evaluation.

1. When the deployed tether length is short, the satellite's nutation motion has a lower frequency than the tether swing motions. Thus, the single degree-of-freedom swing motion 
representation of the tether, attached to a 3-dimensional rigidbody satellite, is good enough for tether modeling. In addition, since the mass of the short tether can be negligible, the mass-less tether model, used in $\S 3$, is the simplest representation for simulation.

2 . As the tether is deployed, the fundamental natural frequency of the tether's swing motion coincides with the nutation motion of the satellite. Then the nutation motion gets higher frequencies than some of the tether's transverse vibration modes. At this point, the simulation model with a few mass points along the tether is necessary to represent the transverse vibrations of the tether. However, the axial deformation of the tether does not have to be considered, since the stiffness in the axial direction is significantly higher than the transverse vibration modes. Therefore, there will be no significant couplings with other dynamics.

3. Since the entire system is spin stabilized, the effect of the tether vibrations with respect to the satellite will be kept small if the rapid actuation is properly avoided. In this case, the entire system will be modeled as a single rigid-body. This simplification will enable long-term navigation simulations where the system is coupled with orbital dynamics.

Finally, in the tests on the ground before flight, the conditions for the tests will be determined based on the mission sequence designed in the simulations. In the conventional satellite, the philosophy is often described as "Test as you fly, fly as you test." However, the hardware tests on the ground are not feasible for the large and flexible structural system like space tethers. Therefore, the verification philosophy will be "Run simulation as you fly, fly as you run simulation."

\section{Conclusion}

This paper first stated that the difference of the simulation objectives between conventional research and nano-satellite development. Since the rapid simulations are needed, this paper clarified the simplest possible analytical model to capture a failure mode in each tether deployment phase. In future work, the actual parameters of FORESAIL-1 Cubesat will be substituted in the analytical model, and the frequency domain criterion will be quantitatively visualized. In addition, an inextensional tether model will be developed for simulating the closed-loop attitude control responses during and after the tether deployment.

\section{ACKNOWLedgments}

The authors acknowledges all the members involved in the development of FORESAIL CubeSats. The authors especially appreciate Nemanja Jovanovic, Bagus Riwanto, and Petri Niemelä at Aalto University for their significant contributions in the FORESAIL CubeSat missions. The Finnish Centre of Excellence in Research of Sustainable Space is developing and will launch FORESAIL-1. The Centre is funded through the Academy of Finland with grant numbers $312351,312356,312357,312358$, and 312390 .

\section{REFERENCES}

[1] E. M. Botta, I. Sharf, and A. K. Misra, "Contact dynamics modeling and simulation of tether nets for space-debris capture," Journal of Guidance, Control, and Dynamics, vol. 40, no. 1, pp. 110-123, 2017.

[2] Y. Ishige, S. Kawamoto, and S. Kibe, "Study on electro- dynamic tether system for space debris removal," Acta Astronautica, vol. 55, no. 11, pp. 917-929, 122004.

[3] B. C. Edwards, "Design and deployment of a space elevator," Tech. Rep. 10, 2000. [Online]. Available: www.elsevier.com/locate/actaastro

[4] P. Janhunen and A. Sandroos, "Simulation study of solar wind push on a charged wire: basis of solar wind electric sail propulsion," Tech. Rep., 2007. [Online]. Available: www.ann-geophys.net/25/755/2007/

[5] G. Mengali, A. A. Quarta, and P. Janhunen, "Electric sail performance analysis," Journal of Spacecraft and Rockets, vol. 45, no. 1, pp. 122-129, 2008.

[6] I. Iakubivskyi, P. Janhunen, J. Praks, V. Allik, K. Bussov, B. Clayhills, J. Dalbins, T. Eenmäe, H. Ehrpais, J. Envall, S. Haslam, E. Ilbis, N. Jovanovic, E. Kilpua, J. Kivastik, J. Laks, P. Laufer, M. Merisalu, M. Meskanen, R. Märk, A. Nath, P. Niemelä, M. Noorma, M. R. Mughal, S. Nyman, M. Pajusalu, M. Palmroth, A. S. Paul, T. Peltola, M. Plans, J. Polkko, Q. S. Islam, A. Reinart, B. Riwanto, V. Sammelselg, J. Sate, I. Sünter, M. Tajmar, E. Tanskanen, H. Teras, P. Toivanen, R. Vainio, M. Väänänen, and A. Slavinskis, "Coulomb drag propulsion experiments of ESTCube-2 and FORESAIL-1," Acta Astronautica, 2019.

[7] M. Palmroth, J. Praks, R. Vainio, P. Janhunen, E. K. J. Kilpua, N. Y. Ganushkina, A. Afanasiev, M. Ala-Lahti, A. Alho, T. Asikainen, E. Asvestari, M. Battarbee, A. Binios, A. Bosser, T. Brito, J. Envall, U. Ganse, H. George, J. Gieseler, S. Good, M. Grandin, S. Haslam, H. P. Hedman, H. Hietala, N. Jovanovic, S. Kakakhel, M. Kalliokoski, V. V. Kettunen, T. Koskela, E. Lumme, M. Meskanen, D. Morosan, M. R. Mughal, P. Niemelä, S. Nyman, P. Oleynik, A. Osmane, E. Palmerio, Y. PfauKempf, J. Peltonen, J. Plosila, J. Polkko, S. Poluianov, J. Pomoell, D. Price, A. Punkkinen, R. Punkkinen, B. Riwanto, L. Salomaa, A. Slavinskis, T. Säntti, J. Tammi, H. Tenhunen, P. Toivanen, J. Tuominen, L. Turc, E. Valtonen, P. Virtanen, and T. Westerlund, "FORESAIL-1 cubesat mission to measure radiation belt losses and demonstrate de-orbiting," 52019. [Online]. Available: http://arxiv.org/abs/1905.09600 http://dx.doi.org/10.1029/2018JA026354

[8] A. Slavinskis, U. Kvell, E. Kulu, I. Sünter, H. Kuuste, S. Lätt, K. Voormansik, and M. Noorma, "High spin rate magnetic controller for nanosatellites," Acta Astronautica, vol. 95, no. 1, pp. 218-226, 22014.

[9] M. R. Mughal, J. Praks, R. Vainio, P. Janhunen, J. Envall, A. Näsilä, P. Oleynik, P. Niemelä, A. Slavinskis, J. Gieseler, N. Jovanovic, B. Riwanto, P. Toivanen, H. Leppinen, T. Tikka, A. Punkkinen, R. Punkkinen, H.-P. Hedman, J.-O. Lill, and J. Slotte, "Aalto-1, multi-payload CubeSat: In-orbit results and lessons learned," Acta Astronautica, 1 2021. [Online]. Available: https://linkinghub.elsevier.com/retrieve/pii/S0094576520307190

[10] J. P. Praks, M. R. Mughal, and et al., "Aalto-1, multipayload CubeSat: design, integration and launch," Acta Astronautica, no. In press, 2021.

[11] M. L. Psiaki, P. M. Kintner, and S. P. Powell, "Rapid Energy Dissipation in a Yo-Yo-Type Wire Boom Deployment System," Journal of Guidance, Control, and Dynamics, vol. 23, no. 3, pp. 483-490, 2000.

[12] D. Haraguchi, H. Sakamoto, Y. Shirasawa, and O. Mori, 
"Design criteria for spin deployment of gossamer structures considering nutation dynamics," in AIAA Guidance, Navigation, and Control Conference, 2010.

[13] J. A. Banik and C. H. Jenkins, Testing Large Ultra-Lightweight Spacecraft, J and J, Eds. Reston ,VA: American Institute of Aeronautics and Astronautics, Inc., 9 2017. [Online]. Available: https://arc.aiaa.org/doi/book/10.2514/4.104657

[14] Y. Miyazaki, Y. Shirasawa, O. Mori, H. Sawada, N. Okuizumi, H. Sakamoto, S. Matunaga, H. Furuya, and M. Natori, "Conserving finite element dynamics of gossamer structure and its application to spinning solar sail "IKAROS"," in Collection of Technical Papers - AIAA/ASME/ASCE/AHS/ASC Structures, Structural Dynamics and Materials Conference, 2011.

[15] H. Sakamoto, Y. Miyazaki, and O. Mor, "Transient dynamic analysis of gossamer-appendage deployment using nonlinear finite element method," Journal of Spacecraft and Rockets, vol. 48, no. 5, 2011.

[16] H. Seppänen, S. Kiprich, R. Kurppa, P. Janhunen, and E. Hæggström, "Wire-to-wire bonding of $\mu \mathrm{m}$-diameter aluminum wires for the Electric Solar Wind Sail," $M i$ croelectronic Engineering, vol. 88, no. 11, pp. 32673269, 112011.

[17] B. Wie, Space Vehicle Dynamics and Control, Second Edition. Reston, VA: American Institute of Aeronautics and Astronautics, 1 2008. [Online]. Available: http://arc.aiaa.org/doi/book/10.2514/4.860119

[18] M. J. Balas, "Active control of flexible systems," Journal of Optimization Theory and Applications, vol. 25, no. 3, pp. 415-436, 7 1978. [Online]. Available: http://link.springer.com/10.1007/BF00932903

\section{BIOGRAPHY}

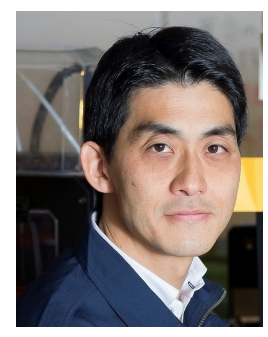

Hiraku Sakamoto received his Ph.D. degree in Aerospace Engineering Sciences from University of Colorado at Boulder, USA. He is currently an Associate Professor, Department of Mechanical Engineering, Tokyo Institute of Technology, Japan. Between 2019-2020, he was a Visiting Scholar at Aalto University, Finland, hosted by Space Technology Group, Department of Electronics and Nanoengineering where he joined the development of the FORESAIL CubeSat.

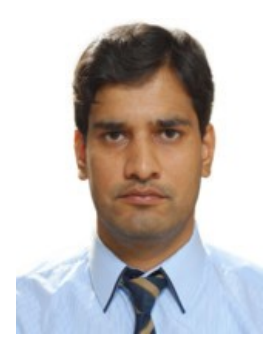

Muhammad Rizwan Mughal received his Ph.D. in Electrical, Electronics and Communications Engineering from Politecnico di Torino, Italy in 2014. He is a Postdoctoral Researcher at Aalto University, Finland, and an Assistant Professor at Institute of Space Technology, Islamabad, Pakistan. He has been a lead engineer and researcher in hardware system design for FORESAIL and Aalto satellites. His research interests include plug and play design of nanosatellites, inter and intra satellite communication systems, and satellite testing.

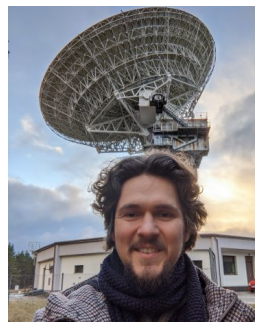

Andris Slavinskis received a Ph.D. degree in Physics from the University of Tartu on ESTCube-1 attitude determination and control. He is currently a Postdoctoral Researcher at Aalto University, Finland, and an Associate Professor at Tartu Observatory, University of Tartu, Estonia. He is interested in small and autonomous missions to asteroids and comets, mission design and simulations, software development and the electric solar wind sail.

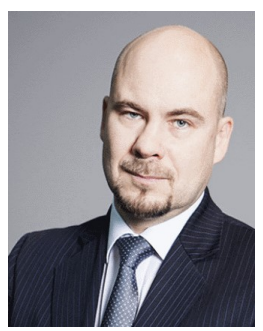

Jaan Praks is an Assistant Professor at Aalto University, and has extensive experience of CubeSat missions developed at educational institutes. He was project lead on both the Aalto-1 and Aalto2 CubeSat projects. The team led by Prof. Praks is the principal developer of the hardware and engineering solutions for the FORESAIL CubeSat missions.

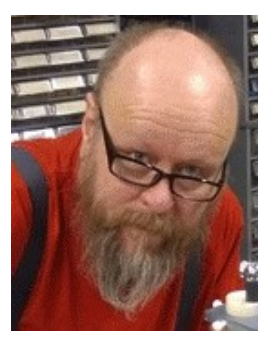

and a slip-ring.

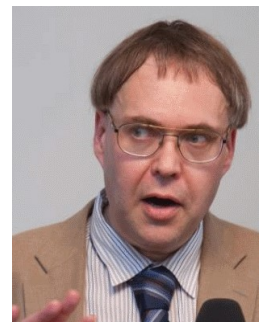

space research.
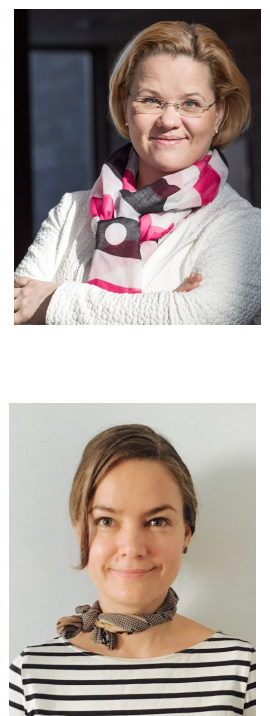

Petri Toivanen is a researcher, Ph.D., in Space Research Unit, Finnish Meteorological Institute, Helsinki, Finland. He is in charge of the development lead for propulsion mission device aboard FORESAIL CubeSats. He especially leads the development of mechanical systems for the tether deployment, including a thin and long tether, a tether reel-in/out mechanism, launch locks,

Pekka Janhunen leads the propulsion team at the Finnish Meteorological Institute for the FORESAIL missions. The electric solar wind sail, or e-sail for short, is a propulsion invention made in 2006 at the Kumpula Space Centre by Pekka Janhunen. The plasma brake application of the e-sail invention plays a primary role in controlled de-orbit of spacecraft, a major factor of sustainable

Minna Palmroth is the director of the Centre of Excellence. She is a Professor of computational space physics in a joint professorship between the University of Helsinki and the Finnish Meteorological Institute. She leads the Modelling team, responsible for providing numerical simulation and modelling tools to assist with the interpretation of the FORESAIL mission data.

Emilia Kilpua is an Associate Professor at the University of Helsinki, leading the Observations team. Her team's primary focus is providing observational information on solar eruptions and related particle acceleration, solar wind structures and their effects in the Earth's radiation environment, and also providing resources for coronal and interplanetary 
modelling. She is a current holder of an ERC consolidator grant SolMAG developing novel coronal simulations and analysing turbulent solar wind structures.

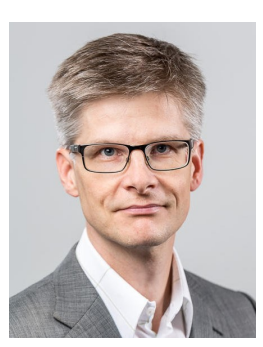

Rami Vainio leads the Space Research Laboratory at the University of Turku. He and his team, including researchers also from the Department of Future Technologies, bring in important experience of building CubeSat mission devices. The Instruments team will be in charge of design and construction of the main scientific payloads aboard the FORESAIL missions. 